\title{
AUTONOMOUS MODELING OF PIPES WITHIN POINT CLOUDS
}

\author{
Mahmoud Ahmed*, Carl T. Haas, and Ralph Haas \\ Department of Civil and Environmental Engineering, \\ University of Waterloo, Waterloo, ON, Canada \\ *Corresponding author (mfouad@uwaterloo.ca)
}

\begin{abstract}
Three-dimensional models are in increasing demand for design, maintenance, operations and construction project management. Point-clouds are the main output of automatic data collection using laser-scanners and photogrammetric technologies. Pipe-works may comprise $50 \%$ of the value of important construction projects such as industrial and research facilities. We have developed a practical and cost-effective approach, based on the Hough-transform and judicious use of domain constraints, to find, recognize and reconstruct/model 3D pipes within point-clouds fully automatically. The developed approach allows pipe growing within a 3D point-cloud. It samples the point-cloud to sequential orthogonal slices, detects pipe's cross-sections within sampled slices and grows the pipe along the cross sections centerlines. It is validated using laser-scanner data from construction of the Engineering-VI building on the University of Waterloo (UW) campus. The system works on a typical laptop. Recognition and localization results are within a few millimeters. The presented approach opens the gate to broad applications of pipe-network modeling.
\end{abstract}




\section{AUTONOMOUS MODELING OF PIPES WITHIN POINT CLOUDS}

\section{INTRODUCTION}

Using 3D point-clouds produced by laser scanners for generating as-built information is becoming a standard practice in construction, rehabilitation and facilities maintenance in areas ranging from process plants to historical preservation, but in the commercial sphere the level of automation is still limited, and underlying algorithms are mostly proprietary. Building on foundational research in robotics and machine vision, research on automated as-built generation goes back well over ten years (Kwon et al 2003, McLaughlin et al 2004, Rabbani et al 2005, Brilakis et al 2010, Ahmed et al 2012). Some of the knowledge thereby created has influenced or been adopted in practice. Additional closely related and overlapping research streams have focused on: (1) quality assessment, (2) automated progress tracking, (3) structural health monitoring, and (4) safety (Teizer et al 2007, Park et al 2007, Chi et al 2009, Ahmed et al 2012).

Acquisition of 3D information with structured lighting, laser-scanning, and photogrammetry has led to automated quality assessment of existing infrastructure and construction sites with a focus on flatness, crack detection, and dimensional compliance (Haas et al 1984, Jaselskis et al 2005, Park et al 2007, Ahmed et al 2011a, b). In parallel, research has progressed on the use of two and three-dimensional image data for construction progress tracking (Cheok et al 2000, Abeid et al 2003, Shih and Huang 2006, Teizer et al 2007, El-Omari and Moselhi 2008, Ibrahim et al 2009, Ahmed et al 2011c). Another approach to automated progress tracking is based on automatic recognition of construction elements using a priori information such as3D Building Information Models (BIM) and 2D images (Wu et al 2010), or BIM and 3D images (Bosche and Haas 2008). Reliance of these object recognition approaches on a priori BIM information does impose limitations.

While piping for industrial facilities such as power plants is typically designed in $3 \mathrm{D}$ and then is fabricated and installed according to the design, piping in most other facility designs today is still not represented in 3D models. For most facilities being built today and for almost all of those which are more than a few years old, their wiring conduit runs and small diameter piping are only notionally or schematically represented in plan-view in their associated design files. Their large bore piping and duct work is typically designed with more dimensional specificity, but frequent undocumented field changes lead to installations that are offset from the original designs. Thus, there is a significant need for development of 3D models of recently completed and older existing facilities. Particularly, pipe and conduit, as distinguished by their cylindrical and toroidal forms, are an important class of model elements.

Automatic and semi-automatic reconstruction of pipe-works in 3D within point-clouds generated using photogrammetry has been investigated (Ahmed et al 2011c; Ahmed et al 2012). Automatic recognition however is challenged by the huge number of points per single 3D point-cloud and by the nature of the data. Specifically, the discrete nature of point-clouds make them easier to comprehend by humans, at least for visualization purposes, than to be automatically interpreted by machines due to the lack of internal spatial and semantic relationships or distinctive features. Pipes, conduit and some ducts, while generally cylindrical, challenge automatic recognition due to their variable shapes, diameters, materials, textures, and spatial distribution. Additionally, the occlusions and the spatial arrangement of pipes in different layers or above hung racks cause the output of most scans to include only fragments of the scanned pipe.

Due to such challenges, automatic recognition without a BIM still typically relies on the use of manual input and heuristic techniques to recognize and interpret spatial features, such as edges, two dimensional (2D) elements, and planar surfaces. Recognition and classification of a broad range of construction elements also rely on reference libraries of typical objects, as reported by the commercial software 
vendors. The Hough-transform offers some potential to address these issues which has been recognized by several researchers (Kwon et al 2003, Cheng and Liu 2004, Rabbani et al 2005).

\section{BACKGROUND ON THE HOUGH-TRANSFORM'S APPLICATION IN CIVIL ENGINEERING}

The Hough-transform can be used to recognize parametric features within noisy data. It is usually carried out in steps. The first step is for incremental transform mapping. This is followed by application of a voting rule, and the last step is concerned with finding the shape parameters within the accumulated array of votes. The technique was first introduced to detect straight lines using a parametric representation of the line in an image. In this case, the Hough-transform requires two parameters, the slope and intercept (Hough, 1962), or the length and orientation of the normal vector to the line from the image origin (Duda and Hart 1972). Then, a modified version was presented for extracting 2D curved shapes (Duda and Hart 1972, Kimme et al 1975) and ellipses (Cheng and Liu2004).

In civil engineering, a 2DHough-transform was used for underground pipe detection (Haas 1986). The use of the 3D Hough-transform for the extraction of planar faces from point-clouds was investigated by (Vosselman et al 2001). But the most recent relevant advance has been its generalized application to point-clouds in (Rabbani et al 2005). While this was ground breaking work, its application was severely limited by its computational complexity. Constraints and heuristics must be applied for practical application as described in this paper. Why high dimensional Hough-spaces are not practical is due to the computational complexity required to support them. Generally, for a typical 3D point-cloud with its millions of points, any representation of more than three dimensions will not be practical, because the Hough-transform is a voting technique based on geometric computations. For example, to find the 2D circle passing through a number of points on a perimeter, each Cartesian point will be represented as a circle in Hough-space. The intersections of all these circles will vote for the center of the original circle in Cartesian space. The voting matrix for circles is three dimensional in Hough-space. Working in Houghspace with more than two dimensions will result in more complexity. Further simplification through judicious use of domain constraints is required.

\section{THE PROPOSED TECHNIQUE}

In the vast majority of built facilities, pipe, conduit, and ducts are built in orthogonal directions along the main axes of the building (Figure 1). Searching in planes perpendicular to these axes for standard reference pipe diameters reduces the problem described above to two dimensions. The raw laser-scan is re-sampled to a number of thin-slices (Figure 2), and the points therein are projected onto one face to create a 2D "image". Due to limited scanner stations, only "arcs" of points representing circular pipe cross-sections are available, yet they suffice as described later. A sequential processing of one thin-slice per time is applied to detect the circles of the presumed standard reference radii in each slice using the Hough-transform. In an initial application of this approach to determine feasibility, a case is examined of pipes along one corridor under construction.

The proposed technique for recognizing and reconstructing the cylindrical shapes within the 3D pointcloud can be summarized in the following steps:

1) Resample original point-cloud to a number of thin separated slices. Slices are chosen at a predetermined interval along the $\mathrm{X}, \mathrm{Y}$ and $\mathrm{Z}$ directions,

2) For each slice, apply Hough-transform (Figure 2) and find the circles satisfying the preknowledge available about the diameters and approximate number of pipes,

3) Connect consecutive centers of detected circles, i.e., grow the centerlines one interval at a time, or alternatively, process all slices, then fit straight lines through all circles' centers,

4) Filter out the systematic errors due to slicing tilt as in (Ahmed et al 2013) 
5) Construct the $3 \mathrm{D}$ pipes using computed centerlines and their respective radii,

6) Visualize the pipes for confirmation in $\mathrm{CAD}$ format on original full point-cloud, or alternatively on the selected slices (Figure 3).

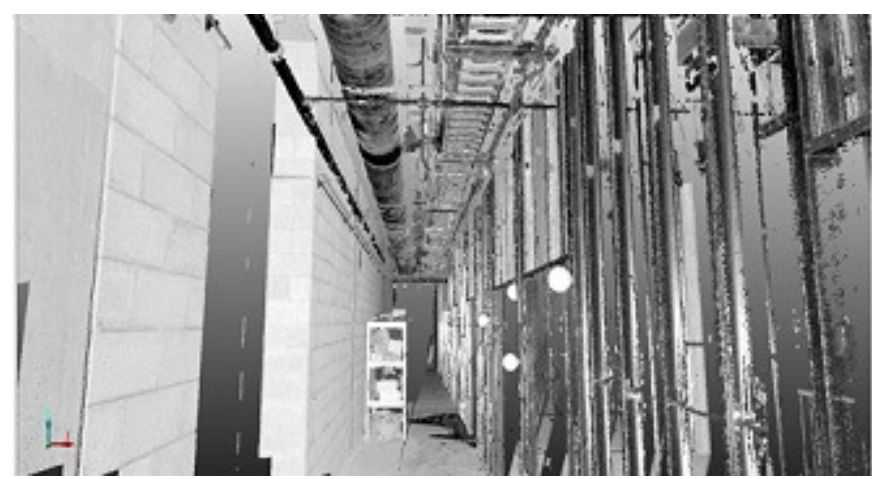

Fig. 1. Laser scan data

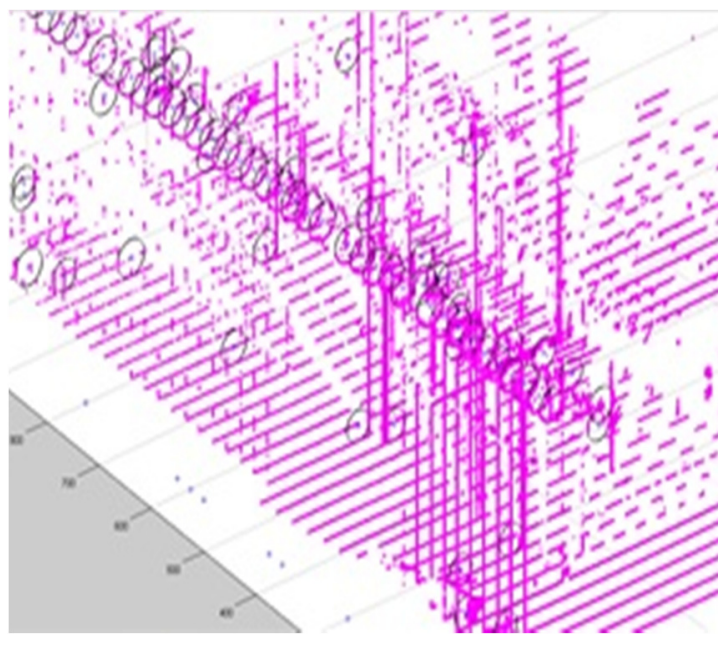

Fig. 2. Detected Circles at sequential cross slices

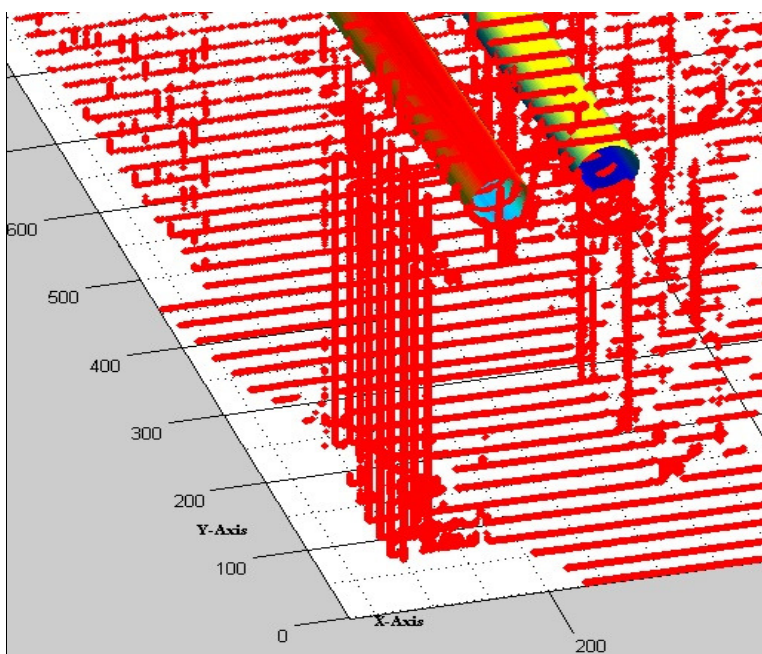

Fig. 3. Generated CAD Pipes

Several reasonable premises are then applied as follows to enable efficient and practical processing in terms of time, speed and hardware resources: 1- some pipes are extended along the corridor under investigation; in a more general case, they may change directions via $90^{\circ}$ elbows, 2- the pipes are hung above the head of the data collecting scanner, 3- assume an approximate range about the number of the pipes in a space and their possible range of discrete diameters for the facility scanned.

After resampling the point-cloud to a number of slices at constant intervals, the second step is to detect the circular cross-sections available in each slice. In the general case, the following equation represents circular pipe cross-sections:

Where:

$$
\mathbf{r}^{2}=(\mathbf{x}-\mathbf{a})^{2}+(\mathbf{y}-\mathbf{b})^{2}
$$

$\mathbf{a}$ and $\mathbf{b}$ are the coordinates of the circle centre,

$\mathbf{x}$ and $\mathbf{y}$ are the coordinates of any point on the circle's circumference, and

$\mathbf{r}$ is radius of the circle. 
Discrete radii are sequentially hypothesized (i.e. pipe outer-diameters available in a reference catalogue are hypothesized to exist in the point-cloud being processed one at a time). Typical diameters in the US for example are 2 and 4 inches. Applying this assumption allows the radius (r) to be treated mathematically as a constant parameter in the equation (1). Accordingly, the transformation to the Houghspace still includes two unknowns, a and b. The relationship between the parametric Hough-space (a, b), and the original Cartesian space (x, y), can be represented by the following equations:

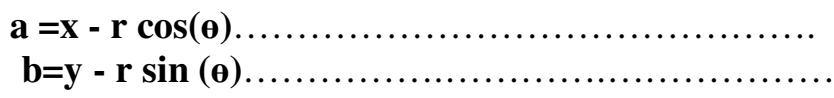

where:

$(\mathbf{a}, \mathbf{b})$ are the circle coordinates in Hough-space, $(\mathbf{x}, \mathbf{y})$ is a point in Cartesian space, which may be on a circle, $\boldsymbol{\theta}$ is a parametric angle, i.e., its value changes in steps from zero to 360 degrees, and $\mathbf{r}$ is the constant radius.

For each slice of point-cloud, each points' coordinates projected onto the $x-y$ plane of the slice are transformed into $a-b$ space. In $a-b$ space, each single point coming from $x-y$ space is represented as a circle. The circle is generated by changing the value of the angle $\theta$ in steps from $0^{\circ}$ to $360^{\circ}$ in equations (2) and (3). Accordingly, all points on a single cross-section in x-y Cartesian space are represented as circles in $a-b$ space. The group of circles in $a-b$ space belonging to cloud points on a circle in $x-y$ space will intersect at one point in Hough-space, in theory.

Normally, the circular cross-sections embedded in one slice generate a number of distinct peaks equal to the number of corresponding pipes, conduit and ductwork present there. However, some errors may occur due to noise. Repeating this process on consecutive slices, and connecting each new circle's center to its corresponding set in the previous slices, using heuristics described later, allows the recognized centerlines of the pipes to be "grown" through the slices, and the pipes to be re-constructed using their radii, despite the noise presence.

After processing all slices, and the centerlines of all radii of pipes are recognized, the re-construction or modeling and the drawing become automatic steps. Then, CAD cylinders in point-cloud coordinates are generated automatically from the circle set's parameters and are stored as text file data or separately as CAD layers (Figure 3).

Theoretically, at least two sections are required to be known to establish the direction. If the length is preknown, the whole pipe can be reconstructed using the two sections and the start point. If the length is unknown, processing the other slices will be required. Applying the developed technique provided more redundant data for the construction of every single pipe. The high redundancy enhanced the line fitting process. The least squares technique estimated the best fitting line that minimize the summation of all the squared values of the residual error. The standard deviation of all cross-section positions along the fitted centerline has been computed as an estimate of final localization precision.

\section{EXPERIMENTAL WORK}

Using the FARO Laser-scanner LS $840 \mathrm{HE}$, data was collected during the construction phases of the E6building on the campus of the University of Waterloo. The scanner provides a theoretical accuracy of $+/-$ $3 \mathrm{~mm}$ at $20 \mathrm{~m}$ distance. The recognition and reconstruction of pipe/duct works installed along the ceiling of each floor from the raw laser-scans were completed fully automatically using the technique explained in the previous sections. Software was developed using Matlab on a typical laptop with the following specs: 8 GB RAM, 1.6 GH Q 720 processor. Thin-slices were selected in perpendicular direction to the pipes. 
Two ranges of discrete diameters, $5-10 \mathrm{~cm}$ and $40-50 \mathrm{~cm}$ at one centimeter intervals, were known in advance and used as input data. It was assumed that there were 1-10 pipes in each diameter range.

Two threshold values were applied based on experience. The total shift in position between any two successive cross-sections, must be within: 1) 2-5 centimeters using low Hough-space granularity, 2 at $1^{\mathbf{o}}$ tilt angle and 5 at $6^{\circ}$ tilt; this one was suitable for diameters around 40-50 centimeters. 2) 3-5 millimeters in case of using high Hough-space granularity; this one was suitable for diameters around 5-10 centimeters. For computations up to centimeter level precision, Hough-space is represented using $800 \times 400(320,000)$ cells (bins). For computations up to millimeter precision, the size of cells requires $32,000,000$ voting bins in the Hough-space corresponding to the same slice. Such increases consume magnitudes more processing cycles. It was found that dealing with original data accuracy in millimeters is enough for localization of the circle center in Hough-space to a precision of \pm 3 to 5 millimeters, provided the systematic errors due to slice tile, are corrected as explained in (Ahmed et al 2013). Figure 4 summarize the accuracy of the investigated technique for the data set used before and after slicing tilt corrections.

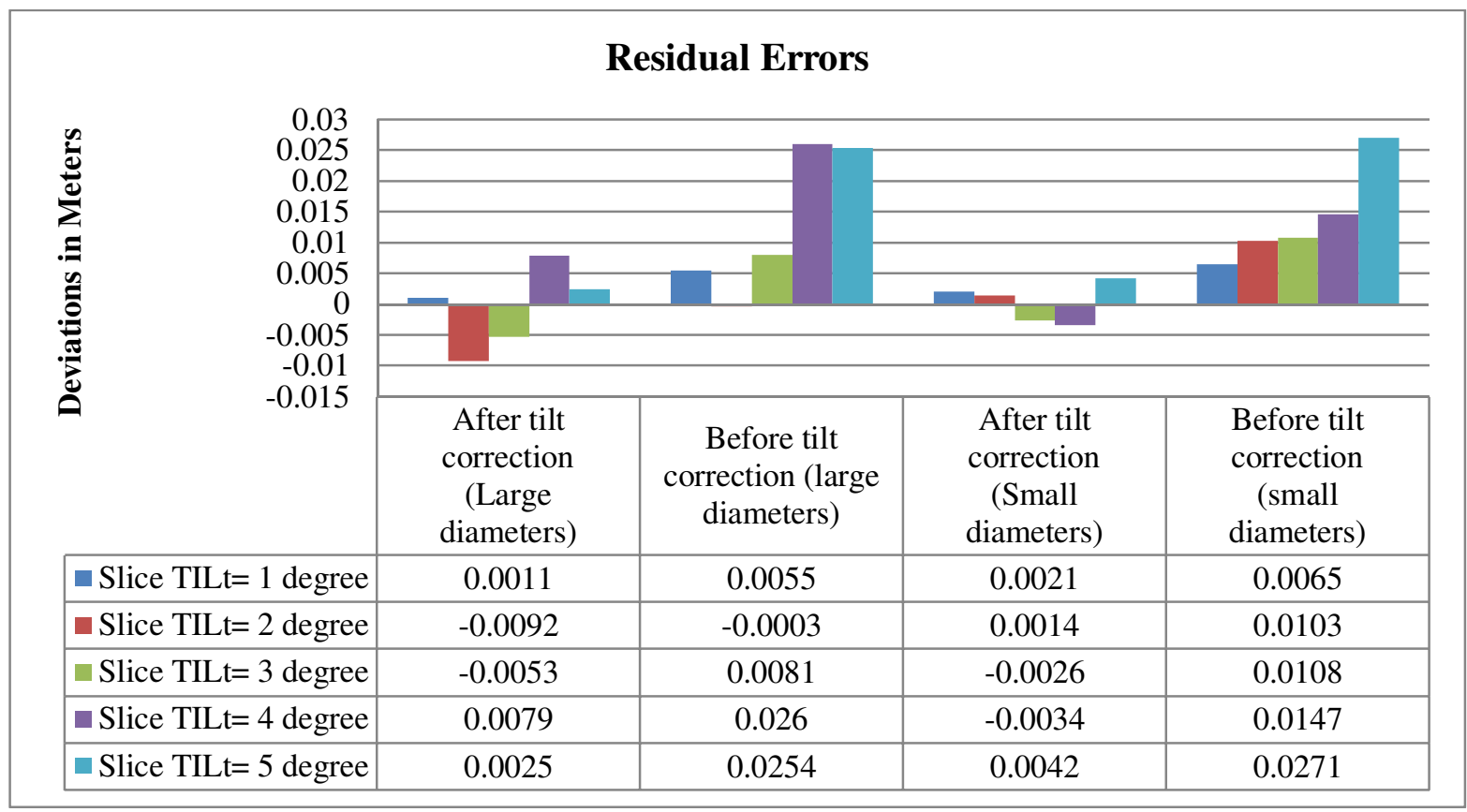

Fig. 4. Final results before and after tilt correction

\section{DISCUSSION OF RESULTS}

For pipe recognition, the use of the pre-knowledge about the installed pipes, and the domain constraints mentioned earlier, reduced computational time and cost, because it enabled the tracking and growing of each group of pipes in one direction in the 3D point-cloud. The process can be repeated in more orthogonal directions depending on the data set under investigation. Key advantages of the technique presented here can be summarized as: 1- It by-passes the need for processing of 5D or greater Houghspace, hence, reducing the overall complexity of the problem from a mathematical point of view 2- One thin-slice is processed per time instead of loading and processing the complete point-cloud, which at present would require special computing facilities, allowing the use instead of a typical laptop or ordinary PC from hardware and software point of view. 3- Using the appropriate pre-knowledge, the use of such an approach can be extended to a broad array of applications and lead to a practical cost-effective solution to 
most of the challenges mentioned earlier. In case of convoluted areas or buildings of complex geometry the user can divide the laser-scan data to several sub-scans and analyze each one separately, then all resulted sub-models can be integrated into one full model.

\section{CONCLUSIONS}

In this research, a new approach for fully automatic detection of cylindrical objects in built facilities was used for recognition and reconstruction of pipes in a point-cloud. The final recognition results are promising under the chosen domain constraints. The level of final localization accuracy is up to a few millimeters. The technique developed and presented in this paper reduces computational complexity. It opens a path toward more generic cylindrical shape recognition, modeling and localization techniques, and a large number of applications as well.

\section{REFERENCES}

Abeid J., Allouche E., Arditi D., and Hayman M. (2003). "Photo-net II: a computer-based monitoring system applied to project management". Automation in construction, 12(5), 603-616.

Ahmed, M., Haas, C., and Haas, R. (2012). "Using Digital Photogrammetry for Pipe-Works Progress Tracking". Canadian Journal for Civil Engineering, CJCE Special Issue on Construction Engineering and Management,issue 9, Vol. 39, 2012.

Ahmed, M., Haas, C., and Haas, R. (2011.a). "Toward Low-Cost 3D Automatic Pavement Distress Surveying: The Close Range Photogrammetry Approach". Canadian Journal for Civil Engineering, Can. J. Civ. Eng. Vol. 38, 2011.

Ahmed, M., Haas, C., and Haas, R. (2013). "Automatic Detection of Cylindrical Objects in Built Facilities".ASCE 2013. (accepted paper)

Ahmed, M., Guillemet, A., Shahi, A., Haas, C., West, J., and Haas, R. (2011.b). "Comparison of PointCloud Acquisition from Laser-Scanning and Photogrammetry Based on Field Experimentation", 3rd International/9th Construction Specialty Conference, Ottawa, Ontario, Canada, June 14-17, 2011

Ahmed, M., Haas, C.T. et al. (2011.c). "Rapid Tracking of Pipe-Works Progress using Digital Photogrammetry", 3rd International/9th Construction Specialty Conference, Ottawa, Ontario, June 14-17, 2011

Bosche, F., Haas, C.T., (2008). "Automated Retrieval of 3D CAD Model Objects in Construction 3D Images". Journal of Automation in Construction, Elsevier, New-York, USA, Volume 17, Issue 4, pp 499512.

Brilakis, I., Lourakis, M., Sacks, R., Savarese, S., Christodoulou, S., Teizer, J., and Makhmalbaf, A. (2010). "Toward Automated Generation of Parametric BIMs based on Hybrid Video and Laser Scanning Data”. Special Issue of the Journal of Advanced Engineering Informatics, 24(4), 456-465.

Chi, S., Caldas, C. H., and Kim, D. Y. (2009). “A Methodology for Object Identification and Tracking in Construction based on Spatial Modeling and Image Matching Techniques". Computer-Aided Civil and Infrastructure Engineering, 24(3), 199-211.

Cheng Z. and Liu Y. (2004). "Efficient Technique for Ellipse Detection using Restricted Randomized Hough transform", Proc. of the International Conference on Information Technology (ITCC'04), Vol.2, pp.714-718.

Cheok, G. S. Stone, W. C. Lipman, R.R. and Witzgall, C. (2000). "LADARs for construction assessment and update", Automation in Construction, 9(5).463-477. 
Duda, R. O. and Hart, P. E., (1972). "Use of the hough transformation to detect lines and curves in pictures".Communincations of ACM 15(1), pp. 11-15.

El-Omari, S., and Moselhi, O. (2008). "Integrating 3D laser scanning and photogrammetry for progress measurement of construction work", Automation in Construction, 18(1), (2008) 1-9.

Haas, C., Shen, H., Phang, W.A., and Haas, R. (1984). "Application of image analysis technology to automation of pavement condition surveys". In Proceedings, International Transport Congress, Montreal, Vol. 5, pp. C55-C74

Haas, C., (1986). “Algorithms to Map Subsurface Ferrous Conductors”, MSc Thesis, Carnegie Mellon University Department of Civil Engineering, Aug. 1986.

Hough, P. V. C., (1962). "Method, and means for recognizing complex patterns". U. S. Patent 3069654.

Ibrahim Y.M., Lukins T.C., Zhang X., Trucco E., Kaka A.P. (2009).“Towards automated progress assessment of work package components in construction projects using computer vision". Advanced Engineering Informatics, 23 (1), 93-103.

Jaselskis, E., Gao, Z., Walters, R.C.,(2005). "Improving transportation projects using laser scanning," Journal of Construction Engineering and Management, 131 (2005) 377-384.

Kimme, C., Ballard, D. and Sklansky, J., (1975). "Finding circles by an array of accumulators". Communications of ACM 18(2), pp. 120-122.

Kwon, S.-W., Bosche, F., Kim,C., Haas, C. T. andLiapi, K. A.(2003). "Fitting Range Data to Primitives for Rapid Local 3D Modeling Using Sparse Range Point-clouds".Automation in Construction, Vol. 13, No. 1.

McLaughlin, J., Sreenivasan, S.V., Haas, C.T. and Liapi, K.A. (2004)."Rapid Human-Assisted Creation of Bounding Models for Obstacle Avoidance in Construction". Journal of Computer-Aided Civil and Infrastructure Engineering, Vol. 19, pp. 3-15.

Park, H. S. Lee, H. M. Adeli, H. Lee, I.(2007). "A new approach for health monitoring of structures: terrestrial laser scanning”. Computer-Aided Civil and Infrastructure Engineering, 22 (1), 19-30.

Rabbani, T and Heuvel, F. van den, (2005). "Efficient Hough transform for automatic detection of cylinders in point clouds". ISPRS WG III/3, III/4, V/3 Workshop "Laser scanning 2005", Enschede, NL.

Shih, N.J. and Huang S.T. (2006)."3D scan information management system for construction management". Journal of construction engineering and management, 132(2), 134-142

Teizer, J. Caldas, C.H. and Haas C.T. (2007). "Real-time three-dimensional occupancy grid modeling for the detection and tracking of construction resources". ASCE Journal of Construction Engineering and Management, 133(11), $880-888$.

Vosselman, G. and Dijkman, S. (2001). "3D Building Model Reconstruction From Point Clouds And Ground Plans". International Archives of Photogrammetry and Remote Sensing, Volume XXXIV-3/W4 Annapolis, MD, 22-24 Oct. 2001.

Wu Y., Kim H., Kim C., and Han S.H. (2010). “Object Recognition in Construction-Site Images Using 3D CAD-Based Filtering". Journal of Computing in Civil Engineering, 24(1), 56-64. 